\title{
Automatic segmentation of calcified plaques and vessel borders in IVUS images
}

\author{
Arash Taki · Zahra Najafi · Alireza Roodaki • \\ S. K. Setarehdan - R. A. Zoroofi • \\ Andreas Konig • Nassir Navab
}

Received: 9 January 2008 / Accepted: 28 May 2008 / Published online: 20 June 2008

(C) CARS 2008

\begin{abstract}
Objective Intravascular ultrasound (IVUS) is a diagnostic imaging technique for tomographic visualization of coronary arteries. Automatic analysis of IVUS images is difficult due to speckle noise, artifacts of the catheter, and shadows generated by calcifications. We designed and implemented a system for automated segmentation of coronary artery IVUS images.

Methods Two methods for automatic detection of the intima and the media-adventitia borders in IVUS coronary artery images were developed and compared. The first method uses the parametric deformable models, while the second method is based on the geometric deformable models. The initial locations of the borders are approximated using two different edge detection methods. The final borders are then defined using the two deformable models. Finally, the calcified regions between the extracted borders are identified using a Bayesian classifier. The performance of the proposed methods was evaluated using 60 different IVUS images obtained from 7 patients.

Results Segmented images were compared with manually outlined contours. We compared the performance of calcified region characterization methods using ROC analysis and
\end{abstract}

A. Taki $(\bowtie) \cdot$ N. Navab

Department of Computer Aided Medical Procedures (CAMP),

TU Munich, Munich, Germany

e-mail: taki@cs.tum.edu

Z. Najafi · A. Roodaki · S. K. Setarehdan · R. A. Zoroofi

Control and Intelligent Processing Center of Excellence,

School of Electrical and Computer Engineering,

College of Engineering, University of Tehran,

Tehran, Iran

A. Konig

Department of Cardiology, Klinikum der Universität München, Medizinische Poliklinik-Campus Innenstadt, Munich, Germany by computing the sensitivity and specificity of the Bayesian classifier, thresholding, adaptive thresholding, and textural features. The Bayesian method performed best.

Conclusion The results shows that the geometric deformable model outperforms the parametric deformable model for automated segmentation of IVUS coronary artery images.

Keywords Border detection - Deformable models · IVUS · Plaque characterization

\section{Introduction}

Intravascular ultrasound (IVUS) is a catheter-based medical imaging technique that is adjunct to angiography in diagnosis for the coronary artery diseases. Using a specially designed ultrasound catheter, it provides real-time tomographic images of the arterial wall that shows the morphological and histological properties of the cross-section of the vessel. Compared to angiography which only depicts a 2D silhouette of the lumen, IVUS not only provides a quantitative assessment of the vessel's wall but also introduces information about the nature of atherosclerotic lesions as well as the plaque shape and size $[1,17]$. Different types of plaques (fibrous, lipid, and calcium) are accumulated between the two layers of the vessel wall (Intima, the inner layer and media-adventitia, the outer layer). The amount of calcium plaques accumulated between the borders is an indicator of the overall plaque burden, and also the degree of calcification will correlate with overall risk of plaque rupture in the coronary arterial tree. So, characterizing the shape and position of the calcified plaques will help the cardiologists to choose the best treatment in order to reduce the risk of operation. The first step towards this important goal is to identify the intima and the media-adventitia borders and to segment the plaque regions. It is a difficult, 
subjective, and time-consuming task to manually perform the segmentation. Therefore, there is an increasing interest in the development of automatic border detection and tissue characterization methods for IVUS images.

Several automatic and semiautomatic border detection techniques are reported in the literature $[7,8,15,18,24]$. Energy minimization of an initial contour either by means of the guided snakes or a cost function was proposed for border detection in IVUS images in [15,24]. Different segmentation methods based on the probabilistic approaches have been proposed in $[7,8]$. Spatial continuity along the sequences of IVUS images was used in [18]. On the other hand, various methods for plaque characterization have been suggested in the past including the use of adaptive thresholding [5] and analyzing the texture based features $[2,20,23]$. The adaptive thresholding method suffers from the normal variations of the gray values in the plaque regions, since it is a crisp method. On the other hand, the texture based methods need computationally expensive procedures while do not provide desirable accuracy.

In order to solve some of these problems, this paper focuses on the development of an automated border detection and tissue characterization algorithm based on the deformable models. In the proposed method, the input images are despeckled by an anisotropic diffusion filter [6]. This is because the speckle noise is an inherent drawback of the ultrasound images making it very difficult to apply any ordinary edge operator to these images. Next, the initial locations of the borders are roughly estimated using different kind of ordinary edge detection methods. The final borders are then identified using both geometric and parametric deformable models. Finally, the calcified regions between the extracted borders are identified using the Bayesian classifier. The performances of the proposed geometric and parametric deformable models are evaluated using 60 different IVUS images obtained from 7 patients. The resulting automatically defined borders are compared to those manually identified by two experts. The statistical analysis of the obtained results shows that the geometric deformable model outperforms the parametric deformable model in extracting the intima and the mediaadventitia borders produce errors in the range of the interobserver variability. After detecting the intima and mediaadvenitia boundaries the region between them is analyzed by the Bayesian classifier for plaque type characterization. The thresholding method is also used in this section. The characterized regions as calcified plaques were also compared to the ones that had been detected by the experts. The results show that the bayesian classifier can effectively characterize the calcified regions with a sensitivity and specificity of 92.674 and $98.5 \%$, respectively.

The rest of the paper is organized as follows. The overall description of the proposed methods is explained in "Methods". The validation procedure of the proposed methods is presented in "Method of Validation". The results of the application of the proposed methods to the data set are presented in "Results". "Discussions" provides a detailed discussion over the results. Finally "Conclusions" concludes the paper.

\section{Method}

Preprocessing

The anisotropic diffusion filter is used to despeckle medical ultrasound images; this method preserves the features and enhances the edges $[6,16]$.

There are some parts of the image in the IVUS frames that are not significant as far as image processing is concerned. These include, for example, the calibration marks and the scale marks. These regions along with the bright catheter ring artifact are replaced by the neighboring gray values. Furthermore, because of the more or less circular structures of the vessels, the planar image in the Cartesian coordinates is converted into the Polar coordinates in order to facilitate the detection steps such as the contour initialization.

The initial contours used in the deformable models have to be as near as possible to the real borders. For detecting an initial contour for intima, the intensity information is used for thresholding. This is done by sweeping the pixels starting from the center of the catheter toward the image borders on a constant angle on a radius $(r)$ while the angle is constant. $I(r$, $\theta$ ) denote the intensity of a pixel located in a radius of $r$ and an angle of $\theta$ in polar coordinate. If $I(r, \theta) \succ T$, where $T$ is the threshold value for intensity, then this pixel will be assumed as a point on the initial border. The value of $\mathrm{T}$ is empirically set to 42 . Figure $1 b$ illustrates the intima's initial contour. For the media-adventitia initial contour the image is first filtered using a $3 \times 3$ low pass filter with a sigma value equal to unity. Then a canny edge detection operator with $\alpha=8$ is applied to each frame in Polar coordinate. An example result is shown in Fig. 1a. After the initial border detection step, the images are converted back to the Cartesian coordinate for further processing.

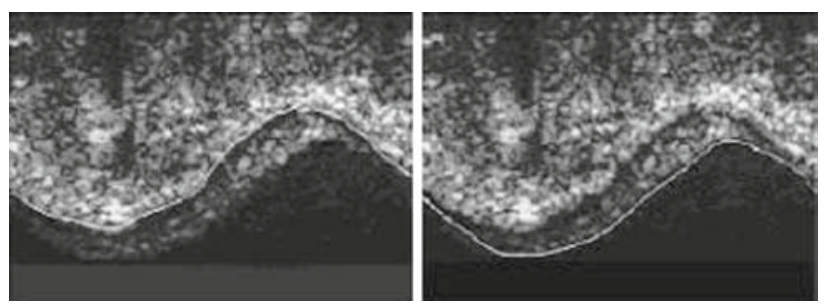

Fig. 1 a Media adventitia initial contour using canny edge detection method. b Intima initial contour using thresholding method 


\section{Border detection}

\section{Parametric deformable models}

Parametric deformable models or snakes are energyminimizing splines guided by the internal constraint forces and influenced by the image forces that pull it towards distinct features (e.g. lines, edges). It was first proposed by Kass et al. [12]. The snake's algorithm is an iterative process used in the image segmentation tasks, particularly for objects whose edges are not well-defined. By taking into account the quantitative energy values for each of the points that make up the snake, the algorithm seeks to find the position of the various snake points that minimize the overall energy of the snake. The energy function for the snake defined by Kass et al. was represented by a vector, $v(s)=(x(s), y(s))$ having arc length, $s$, as parameter:

$E_{\text {snale }}(v)=\int_{0}^{1}\left(E_{\text {int }}(v(s))+E_{\text {ext }}(v(s)) \mathrm{d} s\right)$

$E_{\text {int }}$, represents the internal energy of the snake due to bending or discontinuities, and $E_{\text {ext }}$ is the image force. The image forces can be due to various events, such as lines, edges and terminations [14]. The algorithm involves firstly initializing a set of snake points defined in the preprocessing step from which the iterative process then begins. The parameters of this algorithm were set to the following weights: $\alpha=0.5, \beta=0.5, \gamma=4.2$, and $\rho=1$. These values are defined through an optimization procedure using the results of the border detection method applied to 30 different images (used as the training set) and comparing them to the manually defined borders by experts. The snake algorithm ends when the zero snake points are moved to new positions for five consecutive iterations.

\section{Geometric deformable models}

As mentioned before, the Geometric deformable model scheme is also used for the vessel boundary detection in this work $[10,19]$. As in parametric deformable models the evolution is coupled with the image data to recover object boundaries. Since the evolution is independent of parameterization, the evolving curves and surfaces can be represented implicitly as a level set of a higher dimension function; as a result, topology changes can be handled automatically [11]. Let $u$ be a level set function $u=R^{2} \times[0, \infty] \rightarrow R$ and $c$ is a level set of $u$, such that $c=\left\{x \in R^{2}: u(x, t)=r\right\}, r \in R$. The model is defined as follows:

$$
\begin{aligned}
\frac{\partial u}{\partial t} & =|\nabla u|\left(\operatorname{div}(g(I)) \frac{\nabla u}{|\nabla u|}\right) \\
u(x, 0) & =u_{0}(x)
\end{aligned}
$$

where $u_{0}$ is the initial level set function. The Eq. 2 is equivalent to:

$$
\frac{\partial u}{\partial t}=g(I)(c+k)|\nabla u|+\nabla g \nabla u
$$

where $g(I)$ is the stopping function chosen as

$g(I)=\frac{1}{1+|\nabla \hat{I}|^{2}}$

Its main purpose is to stop the propagation when the evolving front reaches the desired position, i.e. boundaries of the intima or the media-adventitia in the IVUS images. $(\hat{I})$ is a convolved image that ensures the motion of $c$ is less affected by the noise in the image. $K$ is the mean curvature. For the added constant term $c$, we can suppose $c g(I)|\nabla u|$ as an extra speed of the convergence. The last term in Eq. (4) denotes the projection of an attractive force vector on the normal to the moving interface. The narrow band method and fast marching method are two computationally fast and widely used algorithms for the numerical implementation of the geometric deformable models [21]. Instead of computing the evolution of all level sets, which means all the grid points, the narrow band method consists of just updating a small set of points in the neighborhood of the zero level set for each iteration. However, the results of this method depend on the position of the initialized curve/surface. To solve this problem, a new methodology is proposed in this work that can choose the right interface positions, so that the complete boundaries of the intima and the media-adventitia are detected automatically.

\section{Calcified region characterization}

In the previous section, the intima and the media-adventitia boundaries were detected using the geometric and parametric deformable models. The plaque region is defined by extracting the region between these borders. Vessels' plaques are generally composed of calcium, fibrous, and lipid. Calcified regions in IVUS images can be recognized by following characteristics:

- They are usually represented by bright intensity among plaque region.

- As calcium is a hard plaque, the ultrasound beam is not strong enough to penetrate it. Therefore, the calcified region is usually followed by a dense shadow. Two methods for characterizing the calcified regions are studied here. The first is the Bayesian classifier and the other is implemented via setting a threshold value on the pixel intensities. Each of these methods is explained briefly. 


\section{Bayesian classifier}

Bayesian classifier is based on the maximum a posteriori probability, in which the feature vector $X$ is assigned to class $\omega_{j}$ if

$\mathrm{P}\left(\omega_{\mathrm{j}} \mid X\right) \succ \mathrm{P}\left(\omega_{\mathrm{k}} \mid X\right), \quad k=1,2 \ldots M$,

where $M$ is the number of classes and $P$ is the probability. As in most applications the amount of a posteriori probabilities are unknown, its value should be calculated through the Bayes algorithm as follows

$\mathrm{P}(\omega \mid X)=\frac{\mathrm{P}(X \mid \omega) \times \mathrm{P}(\omega)}{\mathrm{P}(X)}$

From the above equation and considering that $P(X)$ is equal for all classes, (6) can be written as

$\mathrm{P}\left(X \mid \omega_{\mathrm{j}}\right) \times \mathrm{P}\left(\omega_{\mathrm{j}}\right) \succ \mathrm{P}\left(X \mid \omega_{\mathrm{k}}\right) \times \mathrm{P}\left(\omega_{\mathrm{k}}\right), k=1,2 \ldots M$

Here, there are two classes, one calcified $\left(\omega_{1}\right)$ and another non-calcified $\left(\omega_{2}\right)$. The probability density function (pdf) of classes is assumed to be Gaussian; therefore, only the values of mean and variance of pdf's should be estimated. These values are attained from the 30 training images, which were collected by the expert from the dataset, and set as follows: $m u_{1}=245, m u_{2}=70, \operatorname{sig}_{1}=20, \operatorname{sig}_{2}=20$. The values of a priori probabilities are achieved experimentally from the 30 training images and are as follows: $\mathrm{P}($ calcified region $)=$ $0.1, \mathrm{P}($ Non-calcified region $)=0.9$.

\section{Thresholding method}

To find an appropriate threshold value in this method, the pixel intensities for the two classes of calcified and noncalcified pixels in the 30 training images are defined and their average value (173.5) is used as the threshold. Therefore, the pixel intensities which are greater than the threshold value are set to be as calcified while the others are set to be non-calcified.

\section{Checking the shadows}

In order to increase the accuracy and reliability of the proposed method, the shadow behind the calcified regions is considered as an important feature. Therefore, for those pixels which are identified as the calcified regions by the thresholding method, the existence of the shadow behind them is examined. In the case of existing shadow, these identified regions are accepted to be calcified region, otherwise they are set back to the non-calcified class. As the IVUS images have circular trait, we first transform the images to the Polar coordinates where the shadows behind the detected calcified regions can easily be checked. For this goal, the average of pixels' intensities, which are placed in the same angle as identified regions, is calculated and the one whose value is below 70 , is accepted to remain as calcified class. The threshold value of 70 was emprically assessed by studying the characteristic of the shadows in the 30 training images.

\section{Method of validation}

\section{Study group}

The IVUS image data set which is used for method validation in this work includes a sequence of IVUS images obtained from 7 different patients. These images, each $500 \times 500$ pixels, were acquired using a 30-MHz transducer at a pullback speed of $0.55 \mathrm{~m} / \mathrm{s}$ and a grabbing rate of 10 frames/s. This process is carried out using the ultrasound system developed by the Volcano therapeutics INC. (model Invision TM, IVG-EE). Sixty frames from each patient have been gathered. The accuracy of the results produced by the proposed methods is determined by comparing them to those produced by the experts. Two experts were asked to manually extract the intima and the media-adventitia borders. The calcified regions are also manually identified by one of the experts.

\section{Accuracy measurements}

For the performance analysis of the proposed algorithms the Average Distance (AD) and the Hausdorff Distance (HD) (the maximum distance) between the automatically identified boundaries and the expert defined ones are determined [9]. These are the most commonly used evaluation parameters proposed and used by other researchers in the past [3,13]. Distance maps serve to compute the differences in positions between automatic and manually traced borders. Such maps encode, for each pixel, $\left(x_{a i}, y_{a i}\right)$ its distance to the closest point on the manual contour:

$$
D(a)=\min _{a i \in \text { border }}\left(\sqrt{\left(X_{a i}-X_{m i}\right)^{2}+\left(Y_{a i}-Y_{m i}\right)^{2}}\right)
$$

Where ai are points on the manually identified contour [3]. Signed distances weigh a value depending on whether the pixel ai is inside or outside the target border. Its mean value detects any bias in curve position (whether detections are systematically bigger or smaller than manual segmentations). We consider these values (absolute distance errors, $D(a)$ ) in millimeter and if we want them in percent(relative distance error) we use $D(a)=100 .(D(a)) /(d(I, O))$, for the origin $O$ the mass center of the manual border and $i$ the point achieving the min in $\mathrm{D}_{a}$. as in reference [9]. For each distance error, its maximum and mean values on the automated contour are the error measures used to assess position accuracy. If PIX denotes the image spatial resolution and ai is any piont on the automatically traced adventitia, then the mean distance 
error(in mm) will be:

$$
M=\underset{a i \in \text { border }}{\operatorname{mean}}(D(a) \cdot P I X)
$$

Also the percentage of the area differences between the manually traced contours and the automated results were calculated. Another parameter for performance analysis used in this work is the William Index (WI) [22], which is the ratio of the average computer-to-observer agreement and the average interobserver agreement [4] as calculated in Eq. 11:

$\mathrm{WI}=\frac{\frac{1}{n} \sum_{j=1}^{n} \frac{1}{D_{0, j}}}{\frac{2}{n-1} \sum_{j} \sum_{j^{\prime} \neq j} \frac{1}{D_{j, j^{\prime}}}}$

where $n$ is the number of observers, $D_{j, j^{\prime}}$ is the average interobserver errors and $D_{0, j}$ is the average error of the proposed method compared to each of the borders defined by expert. The WI values that are close to unity indicate that the difference between our methods and manually detected ones are not significant, so the proposed methods can be considered as accurate as the experts.

\section{Results}

\section{Border detection}

The performances of the border detection methods are investigated. Figure 2 demonstrates the typical intima and the media-adventitia borders identified by each of the proposed automatic methods. The comparative numerical results are demonstrated in Tables 1 and 2. These include the average distances, the HDs and the area difference between the
Fig. 2 a Original IVUS image without calcified regions or stent, b IVUS image with calcified regions, $\mathbf{c}$ and $\mathbf{d}$ results of parametric deformable model and $\mathbf{e}$ and $\mathbf{f}$ are the results of geometric deformable model
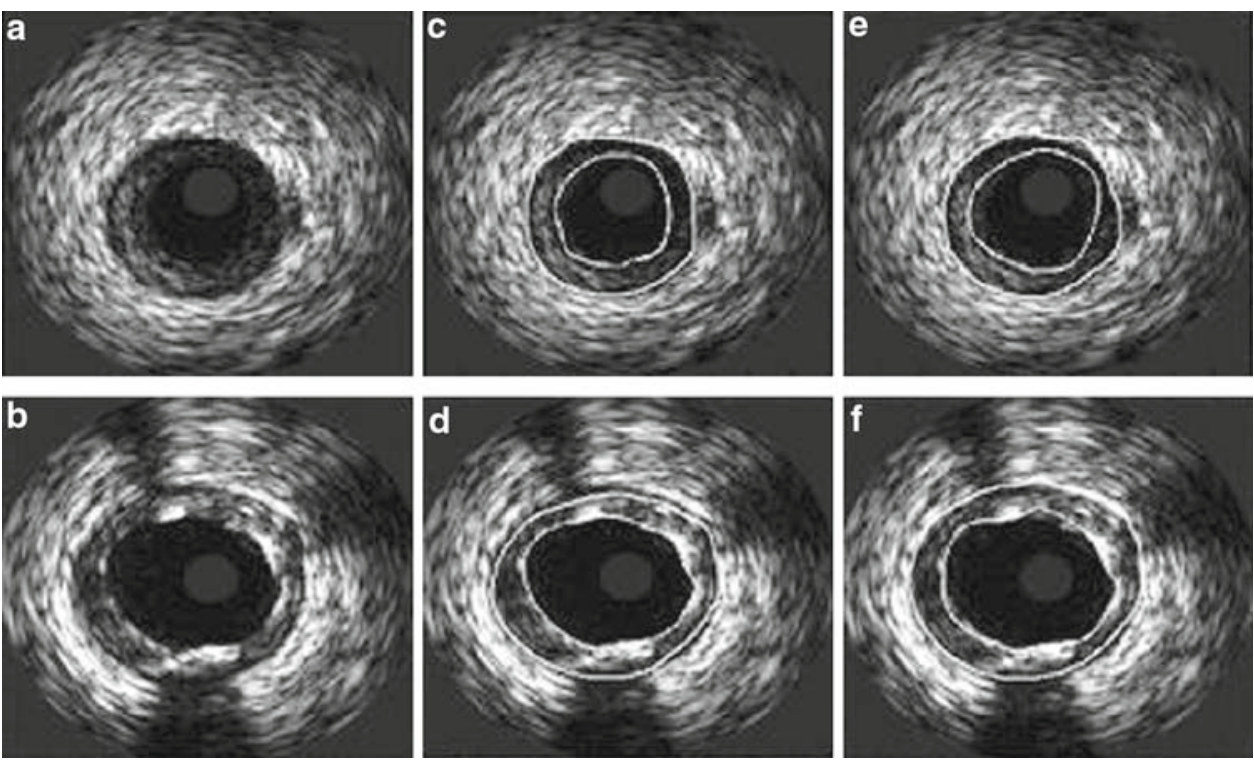

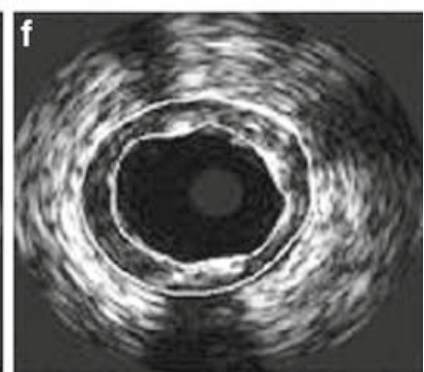

Table $1 A D$ is the average distance, $H D$ is the Hausdorff distance (maximum distance), and Area diff is the absolute difference between the automatically detected and manually traced contour for the Intima border

\begin{tabular}{llll}
\hline Method & Area diff $\left(\mathrm{mm}^{2}\right)$ & HD $(\mathrm{mm})$ & AD $(\mathrm{mm})$ \\
\hline Automatic1 (snake) & $7.5367 \pm 4.6274$ & $0.8817 \pm 0.3115$ & $0.3011 \pm 0.2512$ \\
Automatic2 (level set) & $6.2653 \pm 1.7284$ & $0.7081 \pm 0.2491$ & $0.2031 \pm 0.1502$ \\
Interobserver variability & $5.9312 \pm 1.2573$ & $0.6812 \pm 0.2107$ & $0.1949 \pm 0.0862$ \\
\hline
\end{tabular}

Automaticl is the parametric deformable model method and Automatic 2 is the geometric deformable model method

Table $2 A D$ is the average distance, $H D$ is the Hausdorff distance (maximum distance), and Area diff is the absolute difference between the automatically detected and manually traced contour for the Media-adventitia border

\begin{tabular}{llll}
\hline Method & Area diff $\left(\mathrm{mm}^{2}\right)$ & HD $(\mathrm{mm})$ & AD $(\mathrm{mm})$ \\
\hline Automatic1 (snake) & $6.1232 \pm 1.8332$ & $0.5982 \pm 0.2510$ & $0.3015 \pm 0.0125$ \\
Automatic2 (level set) & $5.0179 \pm 3.7915$ & $0.4531 \pm 0.3120$ & $0.2132 \pm 0.0510$ \\
Interobserver variability & $4.1253 \pm 1.0381$ & $0.5011 \pm 0.1287$ & $0.1158 \pm 0.1013$
\end{tabular}

Automaticl is the parametric deformable model method and Automatic 2 is the geometric deformable model method 

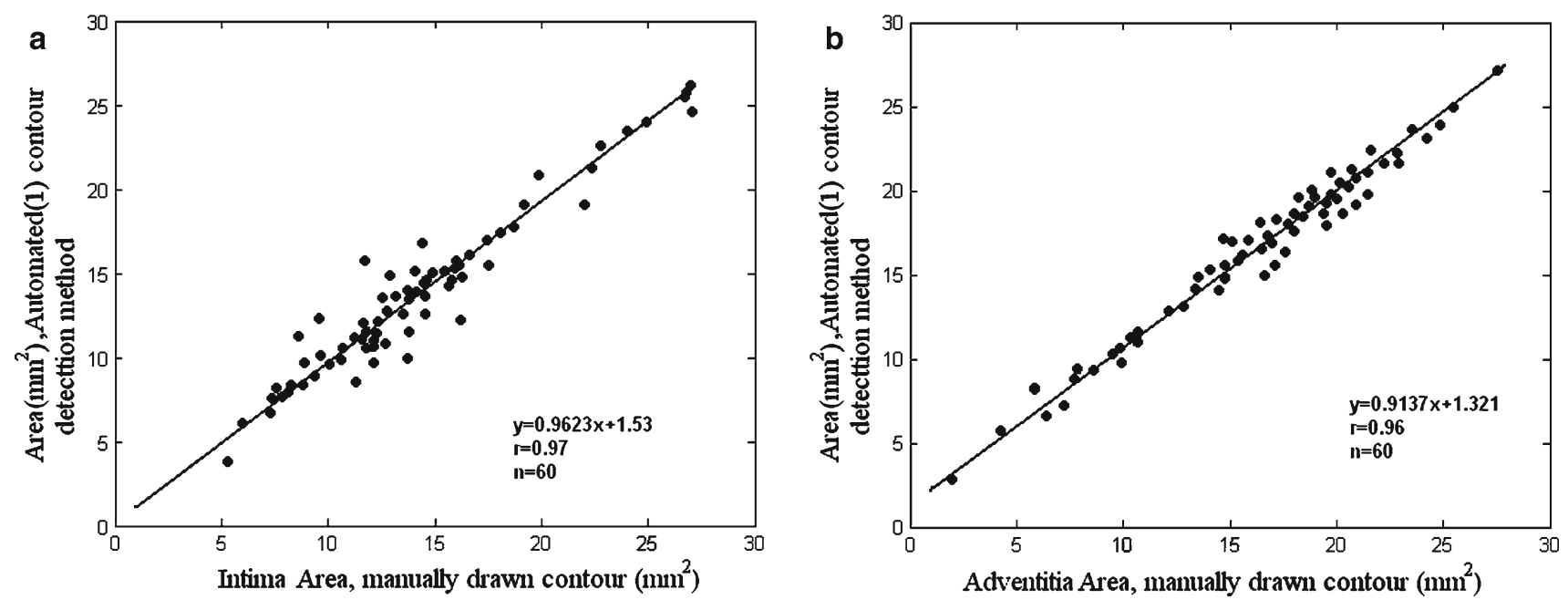

Fig. 3 Comparison of $\mathbf{a}$ Media adventitia and $\mathbf{b}$ intima cross section areas, segmented with geometric deformable models $(n=60)$

manually traced and automatically defined boundaries for the intima and the media-adventitia borders, respectively. Moreover, Fig. 3(a) and (b) illustrate the linear regression analysis between the results of the geometric deformable model and the experts for the intima and for the mediaadventitia borders respectively.

\section{Calcified region detection}

The calcified regions detected by the proposed methods were also compared with those manually detected by the expert.
In order to validate the proposed methods, the sensitivity and specificity of the calcified class were calculated. For this purpose, the true positive (TP), false positive (FP), true negative (TN) and false negative (FN) values were computed. The sensitivity for a class is the percentage of members of that class that are correctly classified by the test. As such, it has to be as high as possible. The specificity for a class is the percentage of members of the other classes that are correctly classified by the test. Table 3 shows these parameters for the two proposed methods and also for other algorithms proposed in the literature. The results of different steps of
Table 3 Comparing the performances of the calcified region characterization methods

Fig. 4 Characterizing calcified region. a IVUS image, b High intensity plaque identification by Bayes algorithm (yellow regions indicates calcified area), c Transformed image in Polar coordinate, $\mathbf{d}$ Removing identified plaques which are not followed by shadow, $\mathbf{e}$ Reconstructed image in Cartesian coordinate (final result), f Manually characterized calcified image by expert

\begin{tabular}{lll}
\hline Classification method & Specificity $(\%)$ & Sensitivity $(\%)$ \\
\hline Bayesian classifier & 98.5 & 92.674 \\
Thresholding method & 83.7 & 74.14 \\
Adaptive thresholding [5] & 88 & 84 \\
Texturural features [2] & 97.2 & 85.5 \\
\hline
\end{tabular}
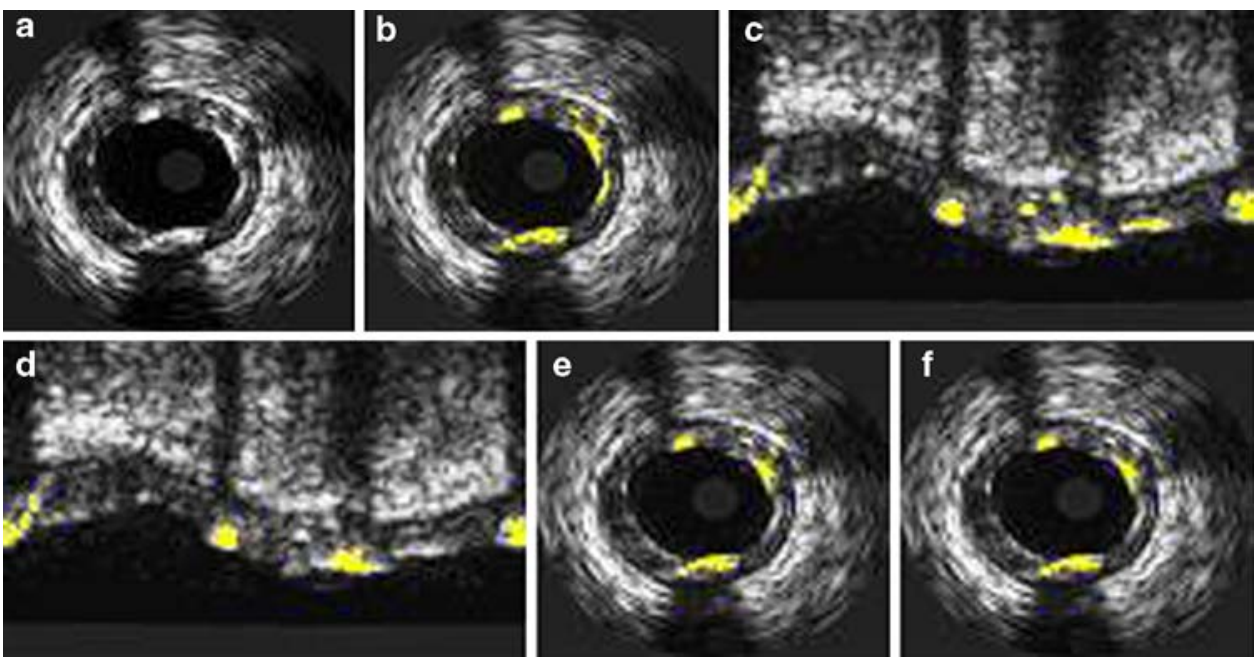


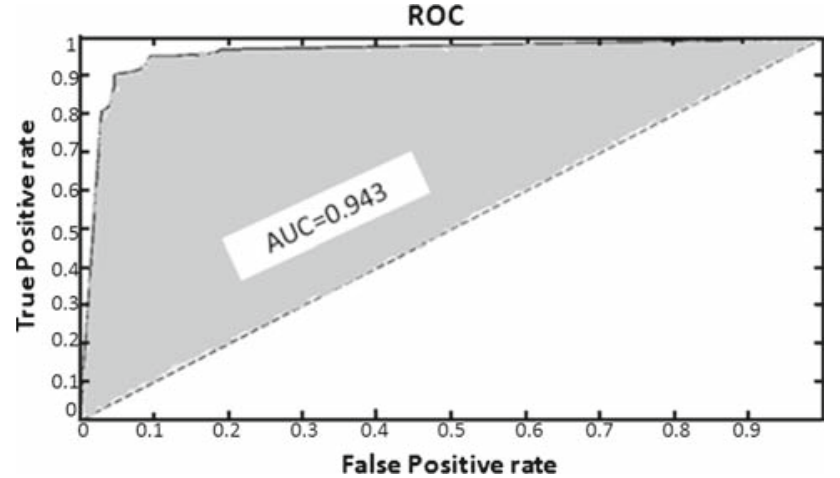

Fig. 5 The receiver operating characteristic (ROC) Curve. The area under the ROC curve is shadowed

characterizing calcified region by means of Bayesian classifier are also demonstrated in Fig. 4.

Another parameter which is used for evaluation of the proposed algorithm is the receiver operating characteristic (ROC) of the Bayesian method, which is more accurate than the thresholding method. This is illustrated in Fig. 5. The area under the ROC curve (AUC) is a reasonable performance statistic for classifier systems. The value of AUC for Bayesian classifier is equal to 0.943 .

\section{Discussions}

At first, the nonlinear anisotropic diffusion filter was applied to the IVUS images. The intraobserver and interobserver variabilities (mean $\pm \mathrm{SD} \%$ ) of the two experts for the intima and the media-adventitia areas have been computed. The intima's intraobserver variability for the first and the second expert was $3.8512 \pm 2.1245 \%(\mathrm{~mm})$ and $4.1307 \pm 1.8579 \%$, respectively. The variability values for the media-adventitia were $3.0565 \pm 3.1645 \%$ for the first expert and $1.96 \pm 0.40 \%$ for the second expert. The values of the interobserver variability are shown in Tables 1 and 2. The results demonstrate that the variability of the two experts is higher for the intima borders. Detection of the intima is more problomatic because of the speckle artifacts and the irregular shape of the intima layer. The linear regression analysis for the geometric deformable model indicates that this method is accurate $(r=0.97, y=0.9623 x+1.53$ for the media-adventitia and $r=0.96, y=0.9137 x+1.3214$ for the intima border detection). The slopes are close to unity, the y-interception confidence interval is close to zero and the correlation coefficient is higher than 0.95. By comparing the AD and HD values from the automatic methods in Tables 1 and 2 the detected borders by the two implemented methods are near the borders detected manually by the experts. Smaller averages and HDs were obtained with the level set method in comparison to the snake algorithm. This shows that the segmentation errors in the second method were small and near the differences between the manual analyses and is powerful for IVUS image segmentation, as we were expecting these values to be significantly higher for the intima than the media-adventitia in both the methods and the experts. We can conclude that, the automatically determined borders corresponded very well with expert manual measurements. The area differences between the borders detected by the two methods show that these values were slightly higher in the parametric deformable model method, but they were still acceptable for the experts. The William index for nonoverlapping areas for the intima in both automated methods were 0.89 and 0.91 , and for media-adventitia 0.90 and 0.93 , respectively. This discrepancy is caused by the lower interobserver variability for the media-adventitia which decreases the value of the William index. As expected, these values also illustrate that the second method (geometric deformable model) is more accurate than the first applied method (the values were closer to unity in second method). In the section of characterizing the calcified regions, the bayesian classifier was applied to IVUS images and the sensitivity of $92.674 \pm 4.6 \%$ was achieved and the specificity was above $98.5 \%$ for all images, in comparison with the regions defined by an expert. In images which exist small and discontinious calcified regions the sensitivity of classifier is reduced to about $85 \%$, but in the case of fragmented or diffused calcified regions this value increases to above $90 \%$.

\section{Conclusions}

In this paper, two automatic methods for defining the calcified regions within the IVUS images were presented. The methods are based on the automatic identification of the intima and the media-adventitia borders by means of the parametric and geometric deformable models.

Border detection and region identification in IVUS images are challenging tasks in medical imaging analysis. Few algorithms have been developed in order to trace the intima and the media-adventitia automatically. In this paper, the preprocessing includes at first a nonlinear anisotropic diffusion filtering that reduces the noise well and preserves the edges of the image. secondly, the initial contours are detected using edge detection methods that makes the deformable model methods automatic. For the geometric deformable model the initial contours are defined to be a distance function for the evolution equation. The level set function in the geometric deformable model was implemented using the narrow band method. This method consists of updating a small set of points in the neighborhood of the zero level set for each iteration.

After detecting the borders with the geometric deformable models method, the calcified regions are identified using the Bayesian classifier and the results are visualized in three dimensions. In our validation methodology, we compared 
the results from the implemented methods with the manual estimation of borders by the two experts. Small variations was observed between the manual and the automated detection of borders, which denotes the accuracy of the methods. The geometric deformable models have some advantages over the parametric ones. First, they are completely intrinsic and, therefore, are independent of the parameterization of the evolving contour. In fact, the model is generally not parameterized until the evolution of the level set function is complete. Second, the intrinsic geometric properties of the contour, such as the unit normal vector and the curvature, can be easily computed from the level set function. This contrasts with the parametric one, where inaccuracies in the calculations of the normal and the curvature result from the discrete nature of the contour parameterization. Besides, because of the leakage of the function, once the curve passes the boundary, it will not be able to pull back to recover the correct boundary. We have solved the problem of the places of the nodes in initialization stage for both methods. The proposed methods have limitations such that they are not accurate where there are side branches or the curvature of the vessel or the catheter. Also, sometimes the frames with the calcified shadowing artifacts will affect on the decision of the methods. For solving the above problems, we suggest to use other frames around this frame for deciding the place of the borders.

Also in this paper, the ability of the Bayesian classifier in characterizing the calcified regions was investigated. The results of our study show that this method has improved the value of sensitivity and specificity in comparison with other algorithms such as the thresholding method or using the texture based features. The Bayes classifier has the advantage of high speed compared to the methods including feature extraction. Therefore, considerable time be saved by using this method.

Acknowledgments The authors would like to thank VOLCANO Company for their help in providing the data set and also their valuable comments.

\section{References}

1. Agostoni P, Schaar JA (2004) The challenge of vulnerable plaque detection in the cardiac catheterization laboratory. $\mathrm{J}$ Kardiovaskulare Med 7:349-358

2. Brunenberg EJL (2005) Automatic IVUS segmentation using feature extraction and snakes Internship report. Dept of Biomedical Engineering, Eindhoven University of Technology, The Netherlands

3. Caselles V, Kimmel R, Sapiro G (1997) geodesic active contours. Int J Comput Vis 22(1):61-79. doi:10.1023/A:1007979827043

4. Chalana V, Kim Y (1997) A methodology for evaluation of boundary detection algorithms on medical images. IEEE Trans Med Imaging 16:642-652. doi:10.1109/42.640755
5. dos Filho E, Yoshizawa M (2004) A study on intravascular ultrasound image processing. J Math Imaging Vis 21:205-223. doi:10.1023/B:JMIV.0000043737.96390.dc

6. Fu S, Ruan Q, Wang W, Li Y (2004) Feature preserving nonlinear diffusion for ultrasonic image denoising and edge enhancement. Trans Eng Comput Technol 2:1305-5313

7. Gil D, Radeva P, Saludes J (2000) Segmentation of artery wall in coronary IVUS Images: a probabilistic approach. Comput Cardiol 4:352-355

8. Gil D, Hernandez A, Carol A, Rodriguez O, Radeva P (2005) A deterministic-statistic adventitia detection in IVUS images. Funct Imaging Model Heart 3504:65-74

9. Gil D, Hernandez A, Rodriguez O, Maura J, Radeva P (2006) statistical strategy for anisotropic adventitia modeling in IVUS. IEEE Trans Med Imaging 25(6):768-777. doi:10.1109/TMI.2006. 874962

10. Han X, Chenyang X, Prince JL (2003) A topology preserving level set method for geometric deformable models. IEEE Trans Pattern Anal Mach Intell 25(6):755-768

11. Han X, Xu C, Prince JL (2001) A topology preserving deformal model using level sets, cvpr, p. 765, 2001 IEEE Computer Society Conference on Computer Vision and Pattern Recognition (CVPR'01), vol 2

12. Kass M, Witkin A, Terzopoulos D (1987) Snakes: active contour models. Int J Comput Vis 1(4):321-331. doi:10.1007/BF00133570

13. Landis JR, Koch GG (1977) The measurement of observer agreement for categorical data. Biometrics 33:159-174. doi:10.2307/ 2529310

14. Lean CCH, Alex KB (2006) An enhanced method for the snake algorithm. Int Conf Innov Comput Inf Control (ICICIC'06) 18:249-258

15. Luo Z, Wang Y, Wang W (2003) Estimating coronary artery lumen area with optimization-based contour detection. IEEE Trans Med Imaging 22(4):48-56

16. Michailovich OV, Tannenbaum A (2006) Despeckling of medical ultrasound images, IEEE transactions on ultrasonics, ferroelectrics and frequency control 53(1):64-48. doi:10.1109/TUFFC. 2006.1588392

17. Nissen SE, Yock P (2001) Intravascular ultrasound clinical application. Am Heart Assoc conf 62:230-242

18. Papadogiorgaki M, Mezaris V, Chatzizisis YS, Kompatsiaris I, Giannoglou GD (2006) A fully automated texture-based approach for the segmentation of sequential IVUS images. Int Conf Syst Signals Image Process 8(2):461-164

19. Suri JS, Liu K, Singh S, Laxminarayan SN, Zeng X, Reden L (2002) Shape recovery algorithms using level sets in 2-D/3-D medical imagery: a state-of-the-art review. IEEE Trans Inf Technol Biomed 6(1):8-28. doi:10.1109/4233.992158

20. Vince DG, Dixon KJ, Cothren RM, Cornhill JF (2000) Comparison of texture analysis methods for the characterization of coronary plaques in intravascular ultrasound images. Comput Med Imaging Graph 24:221-229. doi:10.1016/S0895-6111(00)00011-2

21. Wang H, Ghosh B (2000) Geometric active deformable models in shape modeling. IEEE Trans Image Proc 9(2):302-318

22. Williams GW (1976) Comparing the joint agreement of several raters with another rater. Biometrics 32:619-627. doi:10.2307/ 2529750

23. Zhang X, McKay ChR, Sonka M (1998) Tissue characterization in intravascular ultrasound images. IEEE Trans Med Imaging 17(6):889-899. doi:10.1109/42.746622

24. Zhu H, Liang Y, Friedman MH (2002) IVUS image segmentation based on contrast. In: Processing of SPIE, Durham, NC, USA. pp 1727-1733. Medical Imaging conference, 4684:1727-1733 